ARTIGO ORIGINAL

\title{
Impacto da adição de poliestireno na composição de painéis de bagaço de cana-de-açúcar
}

\section{Impact of the polystyrene addition in composition of sugar cane bagasse panels}

\author{
Ketlin Borges Ferreira ${ }^{1}$ (D) Juliana Ceccato Ferreira ${ }^{1}$ (D), Carlos Rogério Andrade ${ }^{1}$ (D),

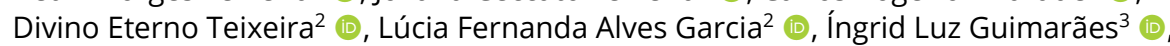 \\ Thaynara Evangelista Silva ${ }^{1}$ (D), Denis Kelington Ferreira Machado ${ }^{1}$ (D) \\ ${ }^{1}$ Universidade Federal de Jataí - UFJ, Jataí, GO, Brasil \\ ${ }^{2}$ Serviço Florestal Brasileiro - SFB, Brasília, DF, Brasil \\ ${ }^{3}$ Engenheira Florestal, Jataí, GO, Brasil
}

Como citar: Ferreira, K. B., Ferreira, J. C., Andrade, C. R., Teixeira, D. E., Garcia, L. F. A., Guimarães, I. L., Silva, T. E., \& Machado, D. K. F. (2020). Impacto da adição de poliestireno na composição de painéis de bagaço de cana-deaçúcar. Scientia Forestalis, 48(126), e2970. https://doi.org/10.18671/scifor.v48n126.20

\begin{abstract}
Resumo
O presente trabalho teve por objetivo avaliar propriedades físicas e mecânicas de painéis compósitos produzidos a partir de bagaço de cana em relação a diferentes proporções de poliestireno (0\%, 10\%, 20\% e $30 \%$ ) e adesivo ureia-formaldeído ( $8 \%, 10 \%$ e 12\%). Os painéis foram prensados na temperatura de $190^{\circ} \mathrm{C}$, pressão de $6,2 \mathrm{MPa}$ por 30 minutos. Para as propriedades físicas avaliou-se teor de umidade, densidade aparente, absorção de água e inchamento em espessura. As propriedades mecânicas analisadas foram módulo de ruptura (MOR), módulo de elasticidade (MOE) pelo ensaio de flexão estática e tração perpendicular. O módulo de elasticidade dinâmico (MOEd) foi determinado, ajustando-se uma equação que melhor representasse sua relação com o módulo de elasticidade estático. Os painéis foram classificados como de baixa densidade, com um valor médio de $0,46 \mathrm{~g} / \mathrm{cm}^{3} \pm 0,015 \mathrm{~g} / \mathrm{cm}^{3}$. Verificou-se uma correlação negativa nas propriedades físicas de teor de umidade, absorção de água e inchamento em espessura com as proporções de poliestireno. As diferentes porcentagens de adesivo não influenciaram significativamente as propriedades físicas, entretanto, as propriedades mecânicas tiveram uma redução quando se utilizou $10 \%$ de adesivo. A partir dos valores do MOEd foi possível ajustar uma equação significativa, com o valor de coeficiente de determinação $\left(R^{2}\right)$ de 0,8051 para estimativa do $M O E$ estático. Painéis produzidos com $8 \%$ de adesivo e 30\% de poliestireno podem ser recomendados na produção de painéis compósitos, entretanto, é indicado o uso de parafina a fim de melhorar as propriedades físicas dos painéis, além da realização de um tratamento químico nas partículas de bagaço de cana antes da confecção dos painéis.
\end{abstract}

Palavras-chave: Resíduos agroindustriais; Plástico; Ureia-formaldeído.

\begin{abstract}
The objective of this study was to evaluate the physical and mechanical properties of composite panels produced from sugarcane bagasse with different proportions of polystyrene $(0 \%, 10 \%, 20 \%$ and $30 \%)$ and adhesive urea-formaldehyde $(8 \%, 10 \%$ and $12 \%)$. The panels were pressed at a temperature of $190{ }^{\circ} \mathrm{C}$, pressure of $6.2 \mathrm{MPa}$ for 30 minutes. For the physical properties, moisture content, apparent density, water absorption and swelling in thickness were evaluated. The mechanical properties analyzed were modulus of rupture (MOR), modulus of elasticity (MOE) by the test of static bending and perpendicular traction. The dynamic modulus of elasticity (MOEd) was determined by fitting an equation that best represents its relation with the static modulus of elasticity. The panels were classified as of low density, with a mean value of $0.46 \mathrm{~g} / \mathrm{cm}^{3} \pm 0.015 \mathrm{~g} / \mathrm{cm}^{3}$. There was a negative correlation in the physical properties of moisture content, water absorption and swelling in thickness with the proportions of
\end{abstract}

Fonte de financiamento: nada a declarar.

Conflito de interesse: Nada a declarar.

Autor correspondente: ketlinlen@hotmail.com

Recebido: 20 julho 2018.

Aceito: 26 julho 2019.

Editor: Francides Gomes Silva Júnior.

(c) (i) Este é um artigo publicado em acesso aberto (Open Access) sob a licença Creative Commons Attribution, que permite uso, distribuição e reprodução em qualquer meio, sem restrições desde que o trabalho original seja corretamente citado. 
polystyrene. The different adhesive percentages did not significantly influence the physical properties; however, the mechanical properties were reduced when $10 \%$ adhesive was used. From the MOEd values it was possible to adjust a significant equation with the coefficient of determination $\left(R^{2}\right)$ of 0.8051 estimate of the static MOE. Panels produced with $8 \%$ adhesive and $30 \%$ polystyrene can be recommended in the production of composite panels. However, the use of paraffin is indicated in order to improve the physical properties of the panels, also a chemical treatment of the bagasse particles before making the panels.

Keywords: Agroindustrial residue; Plastic; Urea-formaldehyde.

\section{INTRODUÇÃO}

De 2010 a 2016 a produção de painéis reconstituídos no Brasil passou de 6,5 para 7,3 milhões de $\mathrm{m}^{3}$, equivalente a um aumento de aproximadamente 10,95\% (Associação Brasileira de Produtores de Florestas Plantadas, 2010; Indústria Brasileira de Árvores, 2017). Com o crescimento da produção de painéis, houve um aumento na demanda por matériaprima, o que leva à procura por outros tipos de materiais de rápido crescimento que possam contribuir de forma quantitativa e qualitativa com o setor. $\mathrm{O}$ emprego de produtos à base de materiais lignocelulósicos (resíduos agroindustriais e fibras vegetais), tem crescido ao longo do tempo, pois vem sendo empregado como fonte de matéria-prima alternativa na produção de chapas de aglomerados devido à diminuição da oferta de madeiras nativas comerciais e de madeira de reflorestamento. Dessa forma, os resíduos gerados pela agroindústria brasileira mostram-se como alternativas ao setor de painéis apresentando-se com vários tipos de resíduos lignocelulósicos com potencialidades de aproveitamento, em especial o bagaço da cana-de-açúcar (Caraschi et al., 2009; Mendes et al., 2012a; Pedreschi, 2009).

O Brasil é um dos maiores produtores de cana-de-açúcar do mundo. Segundo dados da Companhia Nacional de Abastecimento (2018), em 2017 a produção de cana-de-açúcar foi de 635,59 milhões de toneladas. Aliado a essa alta produção há uma grande geração de resíduos, principalmente de bagaço. A União da Indústria de Cana-de-Açúcar (1996) relatou que uma tonelada de cana produz em média $280 \mathrm{~kg}$ de bagaço. De acordo com Luz et al. (2006), a maior parte desse resíduo é utilizada na própria indústria alcooleira e açucareira para a geração de energia, no entanto, o excedente pode ser utilizado em outras aplicações mais nobres como aplicação em painéis compósitos.

Um compósito é constituído de reforço, geralmente sob a forma de fibras e de uma matriz, que é contínua e envolve a outra fase, podendo ser obtido através da combinação de metais, cerâmicas ou polímeros formando um novo material com propriedades diferentes em relação aos seus componentes puros. A grande dificuldade na fabricação de compósitos de materiais poliméricos com fibras naturais está na adesão entre a fibra e a matriz, além da absorção de água pela fibra e sua baixa resistência a ataques químicos e microbiológicos (American Society for Testing and Materials, 2001; Carvalho \& Cavalcanti, 2006; Carvalho Neto et al., 2009).

Polímeros são macromoléculas caracterizadas por seu tamanho, sua estrutura química e interações intra e intermoleculares. São classificados como termoplásticos, termofixos, borrachas e fibras. Os termoplásticos são plásticos que sofrem alterações na estrutura química durante o aquecimento e, após o resfriamento, podem ser moldados novamente, como o polipropileno (PP), o politereftalato de etileno (PET), o polietileno (PE), o policloreto de vinila (PVC) e o poliestireno (PS), os quais representam cerca de $90 \%$ do consumo nacional (Mano \& Mendes, 1999; Minas Gerais, 2009; Spinacé \& De Paoli, 2005). O PS é o pioneiro entre os termoplásticos, entre suas aplicações principais estão embalagens para a indústria alimentícias especialmente copos descartáveis (Banco Nacional de Desenvolvimento Econômico e Social, 2002).

Os autores Geyer et al. (2017), estimaram em 8,3 bilhões de toneladas a quantidade já produzida de plástico no mundo, dado que apenas uma pequena fração destes materiais é reciclada ou reutilizada. Dessa forma os mesmos autores ainda relataram que 6,3 bilhões de toneladas são classificadas como resíduos e $79 \%$ estariam em aterros e na natureza. Esses resíduos poliméricos são considerados um dos grandes problemas ambientais da atualidade, 
pois a maioria possui origem sintética e podem levar dezenas de anos para se degradarem (Bedin, 2009). Diante do exposto, percebe-se a necessidade de buscar novas alternativas de uso para esse material, entre as quais destaca-se sua aplicação em painéis compósitos, devido as propriedades satisfatórias para confecção de painéis, como citadas anteriormente.

Segundo Maciel et al. (2004a), a aplicação do plástico no painel gera um material que possui qualidades peculiares a de um painel convencional. Os autores Alonge et al. (2014), avaliando compósitos produzidos a partir de plástico e casca de arroz um resíduo agroindustrial, relataram que o material desenvolvido apresentou características físicas e mecânicas aceitáveis, indicando uma importância de aprofundamento de estudos sobre esse tipo de material.

De acordo com Mendes et al. (2012a), o adesivo, o qual pode ser substituído em parte ou totalmente por materiais como o PS, é o componente de maior custo na produção dos painéis, o que se torna importante a definição do tipo e da sua quantidade a ser utilizada, no sentido de se buscar uma otimização na relação custo/benefício.

Dessa forma o objetivo deste trabalho foi avaliar as propriedades físicas e mecânicas de painéis compósitos produzidos a partir de diferentes proporções de bagaço de cana e poliestireno proveniente de copos descartáveis com diferentes porcentagens de adesivo.

\section{MATERIAL E MÉTODOS}

\section{Preparo da matéria-prima}

O bagaço da cana-de-açúcar utilizado foi proveniente da empresa Raízen, localizada em Jataí-GO, que tem como principal objetivo a produção de álcool. O PS foi obtido através da aquisição de copos descartáveis transparentes.

Inicialmente, as amostras de bagaço de cana foram secas em estufa a temperatura de $60^{\circ} \mathrm{C}$, durante 4 dias, para redução da umidade inicial. Na sequência, foram transformadas em partículas do tipo sliver as quais foram geradas com a utilização de um moinho do tipo Willey (SL-31) e posteriormente foram peneiradas, sendo utilizadas apenas as partículas que ficaram retidas na peneira de abertura de 35 mesh, como pode ser verificado na Figura 1. O PS foi obtido através da trituração de copos descartáveis por meio da utilização de uma fragmentadora de papéis (Elgin FC-7150C), com partículas variando entre 3 a $6 \mathrm{~cm}$ de comprimento, é interessante uma redução no comprimento dessas partículas para que haja uma maior interação com as partículas de bagaço de cana.

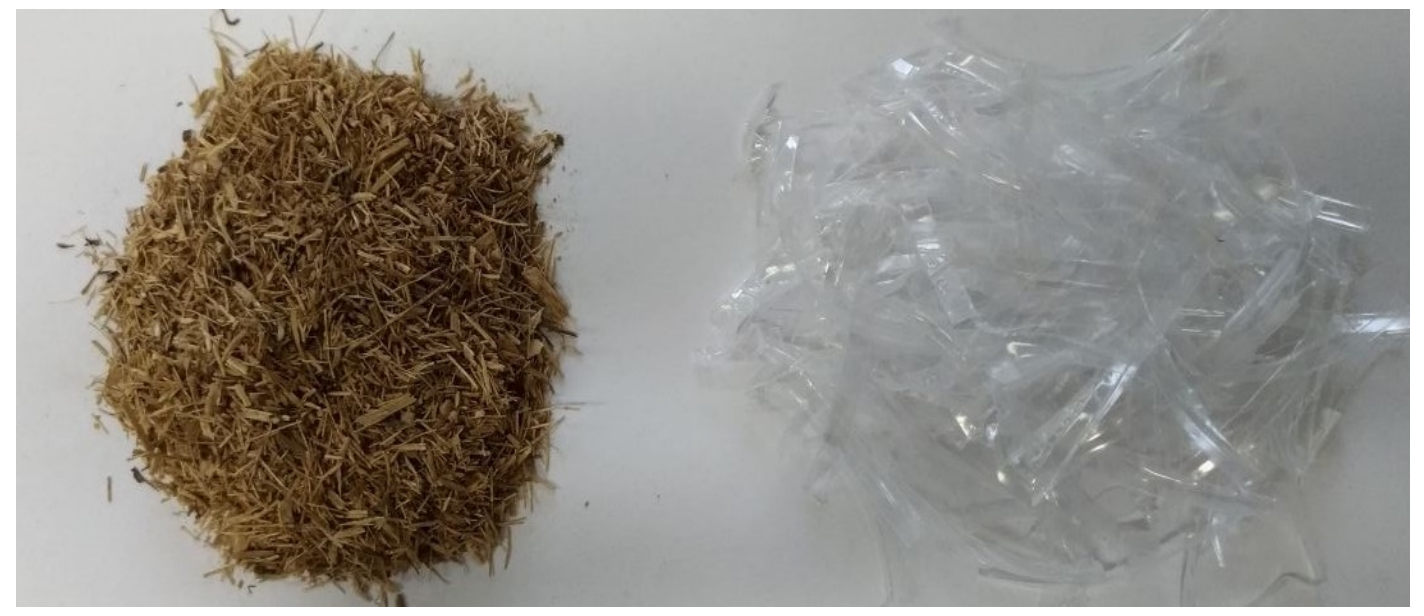

Figura 1. Partículas utilizadas na produção dos painéis compósitos.

A determinação do teor de extrativos totais presentes no bagaço de cana foi realizada de acordo com a norma TAPPI 204 om-88 (Technical Association of the Pulp and Paper Industry, 1997), no Laboratório de Produtos Florestais (LPF), do Serviço Florestal Brasileiro, situado em Brasília-DF. 


\section{Confecção dos painéis}

Os painéis foram confeccionados no Laboratório de Produtos Florestais, da Universidade Federal de Goiás - Regional Jataí. Para a confecção dos painéis compósitos foram utilizadas diferentes porcentagens de adesivo ureia-formaldeído (UF) e diferentes proporções de PS em relação as partículas de bagaço de cana, constituindo os tratamentos dispostos na Tabela 1. Para cada tratamento foram produzidos três painéis com densidade nominal de 0,55 g/cm ${ }^{3}$.

Tabela 1. Delinemento experimental

\begin{tabular}{cccc}
\hline Tratamento & Adesivo (UF) (\%) & Bagaço de cana (\%) & Poliestireno (\%) \\
T1 & 8 & 100 & 0 \\
T2 & 8 & 90 & 10 \\
T3 & 8 & 80 & 20 \\
T4 & 8 & 70 & 30 \\
T5 & 10 & 100 & 0 \\
T6 & 10 & 90 & 10 \\
T7 & 10 & 80 & 20 \\
T8 & 10 & 70 & 30 \\
T9 & 12 & 100 & 0 \\
T10 & 12 & 90 & 10 \\
T11 & 12 & 70 & 20 \\
T12 & 12 & & 30 \\
\hline
\end{tabular}

As porcentagens de UF utilizadas no experimento foram em relação à massa absolutamente seca, das partículas de bagaço de cana. O teor de sólidos do adesivo foi de 64,4\%, com viscosidade de 819 cP, determinado em um viscosímetro Brookfield com um spindler número 18 em uma temperatura de $22,6^{\circ} \mathrm{C}$, gel time de 54 segundos a $130^{\circ} \mathrm{C}$ sem a adição de catalisador e pH de 8,37. As propriedades do adesivo foram obtidas seguindo as normas ASTM D 1490-01, (American Society for Testing and Materials, 2013); ASTM D 1084-97, (American Society for Testing and Materials, 1998); ASTM, D 2471-99, (American Society for Testing and Materials, 1999) e ASTM E70-07, (American Society for Testing and Materials, 2015), respectivamente.

As partículas de bagaço de cana foram secas até atingir 3\% de teor de umidade em base úmida, com auxílio de uma estufa de circulação de ar forçada. Em seguida, foram misturadas manualmente com as diferentes porcentagens de adesivo ureia-formaldeído e PS, sem a adição de catalisador e parafina. Posteriormente foram levadas a uma caixa formadora de colchão, com dimensões de $20 \mathrm{~cm} \times 20 \mathrm{~cm} \times 1,5 \mathrm{~cm}$, para a realização da montagem do colchão e realização da prensagem a uma temperatura de $190^{\circ} \mathrm{C}$ e pressão de $6,2 \mathrm{MPa}$ por um período de 30 minutos, definido após a realização de pré-testes.

Após a produção dos painéis os mesmos foram levados para uma sala de aclimatação com temperatura de $20 \pm 2{ }^{\circ} \mathrm{C}$ e umidade relativa do ar de $65 \%$ até a estabilização da umidade, para posterior confecção dos corpos de prova e determinação das suas propriedades físicas e mecânicas.

Os ensaios físicos e mecânicos dos painéis compósitos foram realizados no Laboratório de Produtos Florestais (LPF), do Serviço Florestal Brasileiro. As propriedades físicas foram realizadas de acordo com a norma NBR 14810-3 (Associação Brasileira de Normas Técnicas, 2002), avaliando-se teor de umidade, massa específica aparente, absorção de água e inchamento em espessura após 2 horas de imersão em água.

Os ensaios de flexão estática pelo qual se determinou o módulo de ruptura (MOR) e módulo de elasticidade (MOE) seguiram uma adaptação da norma EN-310 (British Standards Institution, 1993) que especifica que o comprimento deve ser 20 vezes maior que a espessura da peça. O MOE dinâmico foi obtido por meio da equação 1, disposta a seguir. O equipamento utilizado para obtenção dos dados foi o Stress Wave Timer. 
Equação 1: $M O E_{d}=d * V^{2 *} 1 / g$

Onde:

MOEd = módulo de elasticidade dinâmico $\left(\mathrm{kgf} / \mathrm{cm}^{2}\right)$

$\mathrm{d}=$ densidade $\left(\mathrm{g} / \mathrm{cm}^{3}\right)$

$\mathrm{V}=$ velocidade de propagação da onda $(\mathrm{cm} / \mathrm{s})$

$\mathrm{g}=$ aceleração da gravidade $\left(9,80 \mathrm{~cm} / \mathrm{s}^{2}\right)$.

A determinação da tração perpendicular (ligação interna) seguiu a norma NBR 14810-3 (Associação Brasileira de Normas Técnicas, 2002).

\section{Delineamento experimental}

O experimento foi instalado segundo um delineamento fatorial inteiramente casualizado $3 \times 4$, composto por três porcentagens de adesivo ( $8 \%, 10 \%$ e $12 \%$ ) e quatro porcentagens de PS $(0 \%, 10 \%, 20 \%$ e $30 \%)$ em relação ao bagaço de cana, com três repetições, totalizando 36 unidades amostrais. Os dados foram submetidos aos testes de Lilliefors, para testar a normalidade, e Cochran, para testar a homogeneidade das variâncias. Depois, realizou-se a análise de variância (ANOVA), que foi aplicada para todas as propriedades físicas e mecânicas dos painéis e, quando estabelecido efeito significativo, os mesmos foram comparados entre si, por meio do teste de Tukey, a 95\% de probabilidade. As análises estatísticas foram realizadas com o auxílio do programa STATISTICA 7.0. Para todas as propriedades dos painéis foi aplicada análise de correlação de Pearson.

Após observada correlação significativa entre MOE e MOEd realizou-se uma análise de regressão para estimar uma equação que melhor representasse o comportamento da variável dependente MOE estático em função da variação do MOEd. A escolha se baseou no maior coeficiente de determinação $\left(R^{2}\right)$, menor erro padrão da estimativa e teste $\mathrm{F}$ significativo.

\section{RESULTADOS E DISCUSSÕES}

\section{Caracterização do bagaço de cana}

O teor total de extrativos presente no bagaço de cana foi de $6,35 \% \pm 0,59$, o qual se encontra dentro dos valores citados na literatura de 6,1\% para bagaço de cana sem a realização de um pré-tratamento, de acordo com Gouveia et al. (2009). A presença de extrativos pode influenciar na cura e absorção excessiva do adesivo, resultando assim numa linha de cola de baixa resistência, o que acarreta uma diminuição das propriedades mecânicas dos painéis (Cloutier, 1998; Iwakiri, 2005).

Além dos extrativos, outras propriedades químicas podem interferir na qualidade dos painéis, conforme indicado por Sanchez et al. (2010), os quais relataram que quando comparadas com outras fibras, as de bagaço de cana-de-açúcar apresentam menor resistência a tração, menor módulo de elasticidade e alta absorção de água, devido ao seu alto teor de hemicelulose.

\section{Propriedades físicas dos painéis}

Primeiramente foi realizada a análise de correlação de Pearson entre todas as propriedades físicas e mecânicas dos painéis, a fim de avaliar o efeito de uma propriedade sobre as demais, assim como o efeito da adição de adesivo e das diferentes proporções de PS em relação ao bagaço de cana sobre estas propriedades (Tabela 2). 
Tabela 2. Correlação entre as propriedades físicas e mecânicas dos painéis

\begin{tabular}{|c|c|c|c|c|c|c|c|c|c|c|}
\hline & UF & PS & DA & TU & AA & IE & MOR & MOE & MOEd & TP \\
\hline UF & 1 & & & & & & & & & \\
\hline PS/BC & $-0,11$ & 1 & & & & & & & & \\
\hline DENS & 0,07 & 0,31 & 1 & & & & & & & \\
\hline TU & 0,14 & $-0,93^{*}$ & $-0,38 *$ & 1 & & & & & & \\
\hline AA & 0,06 & $-0,84^{*}$ & $-0,38^{*}$ & 0,86 * & 1 & & & & & \\
\hline IE & $-0,1$ & $-0,72^{*}$ & $-0,17$ & $0,62 *$ & $0,55^{\star}$ & 1 & & & & \\
\hline MOR & 0,16 & $0,34^{*}$ & $0,51 *$ & $-0,37 *$ & $-0,33$ & $-0,34$ & 1 & & & \\
\hline MOE & 0,03 & 0,17 & $0,64^{*}$ & $-0,23$ & $-0,21$ & $-0,08$ & $0,84^{*}$ & 1 & & \\
\hline MOEd & 0,3 & $0,40 *$ & $0,69 *$ & $-0,48^{*}$ & $-0,41 *$ & $-0,29$ & $0,62^{*}$ & $0,63^{*}$ & 1 & \\
\hline TP & $-0,14$ & 0,24 & 0,07 & $-0,33$ & $-0,16$ & $-0,18$ & 0,41 * & 0,22 & 0,19 & 1 \\
\hline
\end{tabular}

*Significativo ao nível de 5\% de significância. UF= Úreia-formaldeido PS/BC=Poliestireno/Bagaço de cana DA=Densidade aparente $\mathrm{TU}=$ Teor de umidade AA=Absorção de água de 2 horas $\mathrm{IE}=$ Inchamento em espessura de 2 horas MOR=Módulo de ruptura MOE=Módulo de elasticidade MOEd= Módulo de elasticidade dinâmico TP=Tração perpendicular.

Verificaram-se elevados valores de correlações negativas, próximos de -1, pela adição do PS com o teor de umidade, absorção de água e inchamento em espessura, o que é esperado, tendo em vista que se busca a obtenção de menores valores para estas propriedades nos painéis. Para o adesivo ureia-formaldeído foram observados baixos valores de correlação.

Observou-se correlação significativa entre o teor de umidade, absorção de água e inchamento em espessura, demonstrando assim que os painéis que apresentam menores valores de teor de umidade tendem a possuir melhor estabilidade dimensional.

Já a densidade aparente apresentou correlação positiva com as propriedades mecânicas MOR, MOE e MOEd. De acordo com Santos et al. (2009), a densidade possui estreita relação com as propriedades mecânicas dos materiais, logo quanto maior o valor da densidade, maior tende a ser a resistência mecânica de um material.

Ao realizar a análise de variância para a densidade aparente dos painéis, verificouse que seus valores não apresentaram diferenças significativas em função das variáveis isoladas de adesivo e de PS, nem da sua interação. A densidade aparente média dos painéis foi de $0,46 \mathrm{~g} / \mathrm{cm}^{3} \pm 0,015$, sendo assim inferior a densidade nominal dos painéis. De acordo com Iwakiri et al. (2004), este comportamento pode ser atribuído, principalmente, à perda de partículas durante o manuseio, em razão das condições laboratoriais de manufatura. Outro fator que pode estar relacionado segundo Moslemi (1974) é o aumento da espessura dos painéis o qual pode ser em função da liberação parcial das tensões de compressão ocorrida logo após o final da prensagem. O mesmo autor menciona ainda que quanto maior a altura do colchão a ser comprimido para uma mesma espessura final, maiores serão as tensões de compressão às quais o painel estará sujeito durante a operação de prensagem. Fato esse que foi observado nos painéis onde a espessura final foi de $2,0 \mathrm{~cm} \pm 0,12$, sendo que a espessura final préestabelecida era de $1,5 \mathrm{~cm}$.

Os painéis foram classificados como sendo de baixa densidade de acordo com a norma CS 236-66 (Commercial Standard, 1968), pois apresentaram valores inferiores a 0,60 g/ $\mathrm{cm}^{3}$. Soares et al. (2017), avaliando painéis produzidos com bagaço de cana em associação com eucalipto, encontrou valores próximos aos relatados nesse trabalho de $0,53 \mathrm{~g} / \mathrm{cm}^{3}$, no qual relata que baixa densidade encontrada nos painéis se refere ao fato do bagaço de cana apresentar uma densidade baixa quando comparada a madeira. Segundo Kelly (1977), quanto maior for a densidade dos painéis, maior será a resistência mecânica, dessa forma os painéis avaliados neste trabalho tendem a possuir baixa resistência mecânica, por apresentarem baixa densidade. Sendo assim os painéis não devem ser indicados para uso estrutural, mas sim para aplicações que não exijam elevadas solicitações mecânicas, podendo se realizar 
testes para verificar suas propriedades em isolamento térmico e acústico, bem como sua utilização em objetos de decoração entre outros (Baldin et al., 2016).

Os valores médios de teor de umidade estão expostos na Figura 2. Houve efeito significativo da interação entre adesivo e PS, para o teor de umidade dos painéis.

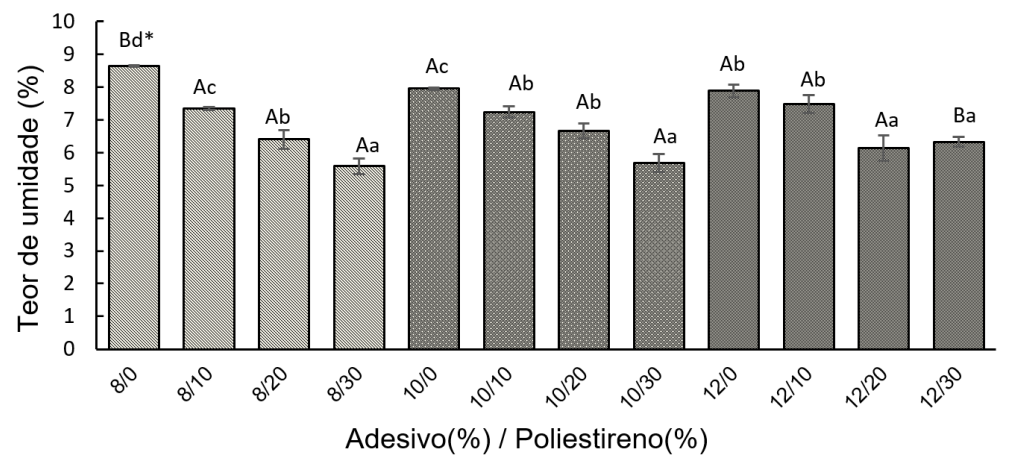

Figura 2. Teor de umidade média dos painéis compósitos de bagaço de cana. *Médias seguidas pela mesma letra minúscula entre porcentagens de PS dentro de cada porcentagem de adesivo e letras maiúsculas entre porcentagens de adesivo dentro de cada porcentagem de PS não diferem significativamente entre si pelo teste Tukey, a 5\% de significância.

Houve uma redução do teor de umidade a medida em que se aumentou a porcentagem de PS nos painéis. Isso se deve ao fato de o PS ser um material hidrofóbico, sendo um fator importante na redução de absorção de água, tendo em vista que na Tabela 2, verificou-se uma correlação positiva do teor de umidade com a absorção de água. Outro fator que influenciou foi o fato de ter reduzido as proporções de bagaço de cana nesses painéis. Os autores Caraschi et al. (2009), também constataram essa tendência avaliando painéis produzidos com casca de arroz e plástico. Os menores valores de teor de umidade foram observados para os painéis que receberam 8 e $10 \%$ de UF, enquanto que a adição de $12 \%$ proporcionou um maior teor de umidade, diferindo significativamente dos demais. Isto pode estar associado a alguma interação negativa em que o maior teor de adesivo causou junto ao poliestireno, afetando a qualidade física do painel.

Para os resultados do ensaio de absorção de água após duas horas de imersão em água fria, houve efeito significativo apenas pela adição do PS, conforme observado na Figura 3. O adesivo não influenciou diretamente nesta propriedade, o que também é relatado por Iwakiri (2005), em virtude da ureia-formaldeído apresentar alta susceptibilidade a degradação hidrolítica na presença de umidade.

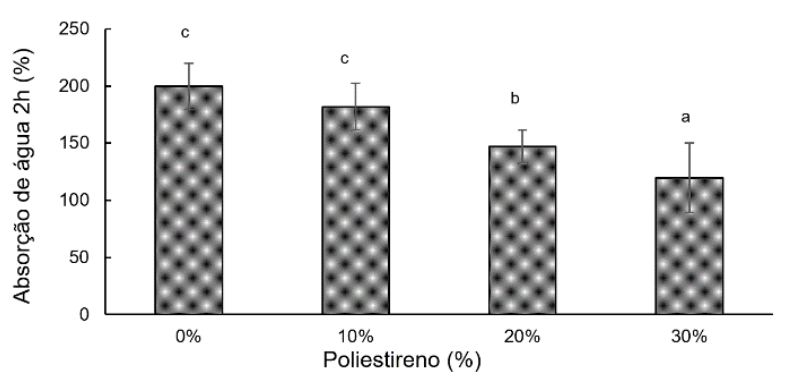

Figura 3. Absorção de água média dos painéis compósitos de bagaço de cana. *Médias seguidas pela mesma letra não se diferem estatisticamente pelo teste Tukey, a 5\% de significância.

Os tratamentos com 0\% e 10\% de PS, não apresentaram diferenças significativas entre si, contudo tiveram as maiores taxas de absorção de água. Nos tratamentos com $20 \%$ e $30 \%$ de PS é possível verificar que a medida que se aumenta as porcentagens dos mesmos nos painéis se tem uma redução da absorção. Entre a adição de 0 para 30\% de poliestireno 
ocorreu uma redução de aproximadamente de $60 \%$ na absorção de água, o que é muito atrativo para a aplicação industrial destes painéis, possibilitando maiores formas de utilização do mesmo, em ambientes com maior contato com umidade.

Os autores Maciel et al. (2004a) verificaram a mesma tendência ao avaliarem painéis confeccionados com Pinus elliottii e diferentes proporções de PS, os quais relatam que isso se deve pelo fato do plástico ser um material hidrofóbico. Uma outra alternativa para reduzir os valores desta propriedade é aplicar parafina juntamente com o adesivo, visto que a parafina também possui propriedade hidrofóbica.

$\mathrm{Na}$ Figura 4, observa-se os valores médios de inchamento em espessura, para duas horas de imersão em água, observando efeito significativo da interação entre as porcentagens de adesivo e PS.

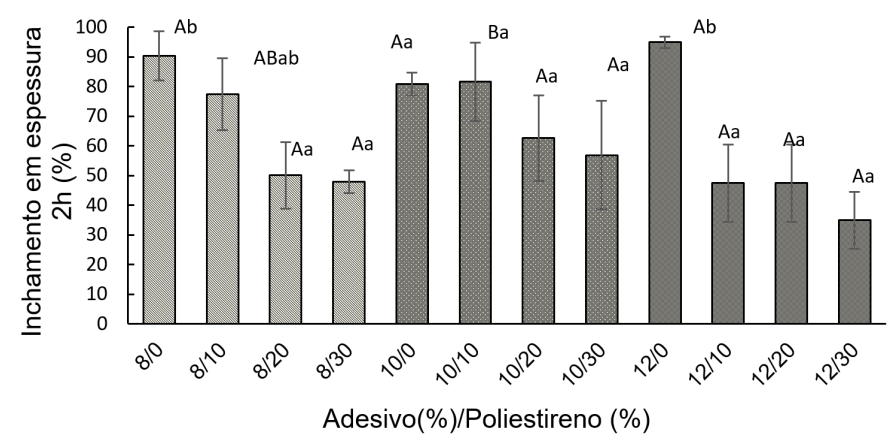

Figura 4. Inchamento da espessura média dos painéis compósitos de bagaço de cana. * Médias seguidas pela mesma letra minúscula entre porcentagens de PS dentro de cada porcentagem de adesivo e letras maiúsculas entre porcentagens de adesivo dentro de cada porcentagem de PS não diferem significativamente entre si pelo teste Tukey, a 5\% de significância.

Semelhante ao observado para a absorção de água, verifica-se na Figura 4, que a medida em que se tem um aumento na porcentagem de PS, ocorre uma redução no inchamento em espessura. Maciel et al. (2004a), relataram que os menores valores de inchamento estão relacionados as maiores porcentagens de plástico, isso se deve pelo fato do plástico ser um material hidrofóbico, como já mencionado.

Os painéis com $0 \%$ de PS, não apresentaram diferença estática entre 8\%, 10\% e 12\% de adesivo. Para os tratamentos com $20 \%$ e $30 \%$ de PS, observou-se que não houve diferença significativa mesmo com as diferentes porcentagens de adesivo, entretanto, menores valores de inchamento foram verificados nos painéis com maior adição de PS e com $12 \%$ de adesivo.

Os altos valores de absorção de água e inchamento estão associados tanto à ação da ureia-formaldeído, que possui baixa resistência à umidade e à ausência de parafina nos painéis, comumente utilizado em painéis comerciais. Neste trabalho optou-se por não utilizar parafina para verificar o real efeito do adesivo e do PS. É importante salientar que o PS pode substituir tanto o adesivo quanto a parafina por apresentar propriedades adesivas e hidrofóbicas. Outro fator que pode estar relacionado com esses altos valores de acordo Sanchez et al. (2010), avaliando compósitos produzidos a partir de fibras de bagaço-de-cana é que quando não se faz o tratamento químico desse material se tem uma maior absorção de água quando comparada com compósitos produzidos a partir de fibras tratadas, isso se deve ao fato de que o tratamento químico das fibras aumenta a interação na interface fibra/matriz, diminuindo os espaços vazios nesta interface e, consequentemente a absorção de água.

\section{Propriedades mecânicas dos painéis}

Os valores médios de MOR e MOE obtidos pelo ensaio de flexão estática estão representados nas Figuras 5, 6, 7 e 8. Tanto para o MOR quanto para o MOE houve efeito significativo isolado das diferentes porcentagens de adesivo bem como das porcentagens de PS adicionadas, não havendo efeito significativo da interação entre eles. 


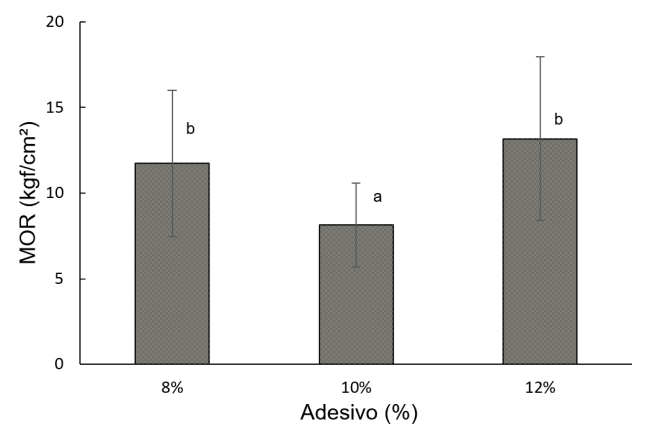

Figura 5. Valores médios de MOR dos painéis compósitos de bagaço de cana. *Médias seguidas pela mesma letra não se diferem estatisticamente pelo teste Tukey, a 5\% de significância.

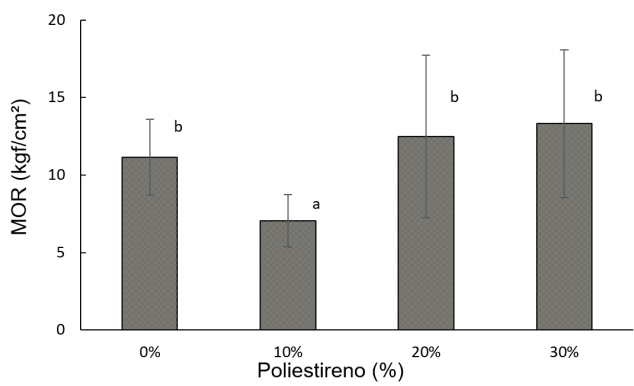

Figura 6. Valores médios de MOR dos painéis compósitos de bagaço de cana. *Médias seguidas pela mesma letra não se diferem estatisticamente pelo teste Tukey, a 5\% de significância.

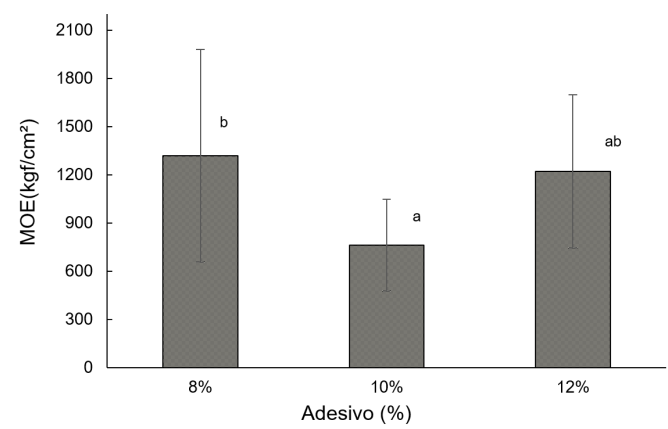

Figura 7. Valores médios de MOE dos painéis compósitos de bagaço de cana. *Médias seguidas pela mesma letra não se diferem estatisticamente pelo teste Tukey, a 5\% de significância.

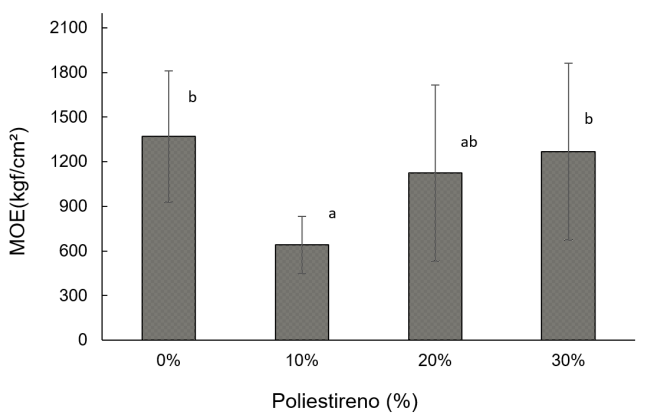

Figura 8. Valores médios de MOE dos painéis compósitos de bagaço de cana. *Médias seguidas pela mesma letra não se diferem estatisticamente pelo teste Tukey, a 5\% de significância.

Conforme pode ser observado na Figura 5, nota-se que não houve diferença significativa entre painéis com adição de $8 \%$ e $12 \%$ de adesivo, que tiveram os maiores valores de resistência mecânica ao ensaio de flexão estática. Na Figura 6, observa-se também que não 
houve diferença significativa entre os painéis com adição de 0\%, 20\% e 30\% de PS, entretanto, quando se adicionou $10 \%$ de PS com o bagaço da cana, reduziu os valores de MOR demonstrando que baixas porcentagens de PS podem influenciar negativamente essa propriedade.

Para o ensaio de MOE, na Figura 7, pode se observar que o painel produzido com $12 \%$ de adesivo não apresentou diferença estatística significativa com os demais tratamentos, com o maior valor de resistência observado para os painéis com adição de $8 \%$ de adesivo. Já na Figura 8, observou-se a mesma tendência que ocorreu para MOR, onde houve uma redução quando foi adicionado $10 \%$ de PS nos painéis, o que já era esperado devido ao MOR e MOE apresentarem alto valor de correlação. E não foi encontrada diferença estatística significativa nos painéis produzidos com 0, 20 e 30\% de PS. De modo geral, os autores Maciel et al. (2004b), avaliando painéis produzidos a partir de Eucalyptus grandis e PS verificaram maiores valores para MOR e MOE à medida que se aumentou a porcentagem de PS nos painéis.

O autor Wu (1999), não encontrou uma tendência definida avaliando diferentes quantidades de adesivo em relação aos valores de módulo de elasticidade e ruptura. Entretanto, Mendes et al. (2012a), observou que a medida em que se aumentava as porcentagens de adesivo se teve um aumento nestas propriedades.

Os baixos valores de MOR e MOE são atribuídos a baixa densidade do bagaço de cana que, de acordo com Mendes et al. (2012a), esse fator implica em uma quantidade maior de partículas para ser colada com a mesma quantidade de adesivo, resultando assim em uma menor quantidade de adesivo por partícula. Já os autores Soares et al. (2017), ao avaliarem painéis produzidos com consorciação de bagaço de cana e eucalipto, relataram que o acréscimo de $1 \%$ desse resíduo no painel, promoveu uma redução de, aproximadamente $86,10 \mathrm{kgf} / \mathrm{cm}^{2}$ para o MOE e de $1,02 \mathrm{kgf} / \mathrm{cm}^{2}$ para o MOR. Como mencionando anteriormente, os baixos valores nestas propriedades podem ser atribuídos também ao alto teor de hemicelulose presente no bagaço de cana. Os valores de MOE e MOR foram inferiores aos pré-estabelecidos pela norma de comercialização ANSI/A208.1 (American National Standard, 1993), 5608,44 e 30,6 kgf/ $\mathrm{cm}^{2}$, respectivamente.

$\mathrm{Na}$ Figura 9, estão os valores médios de tração perpendicular o qual houve efeito significativo das diferentes proporções do adesivo não sendo influenciada diretamente pelo PS.

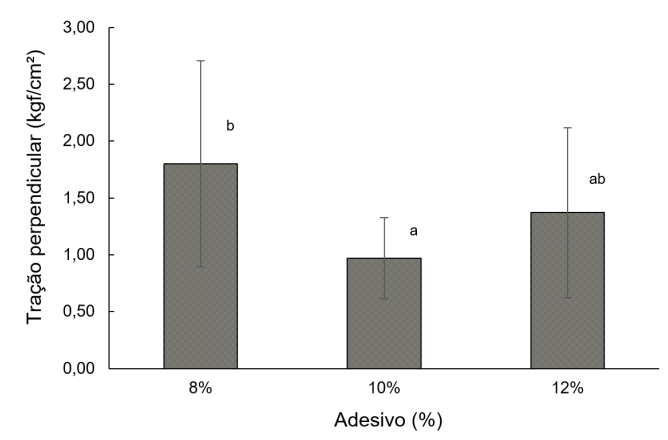

Figura 9. Valor médio de tração perpendicular dos painéis compósitos de bagaço de cana. *Médias seguidas pela mesma letra não se diferem estatisticamente pelo teste Tukey, a 5\% de significância.

De acordo com Guimarães Júnior et al. (2016), a tração perpendicular é uma propriedade que avalia a relação de colagem entre as partículas, por isso houve influência do adesivo nesta propriedade. Dessa forma observou-se que painéis com $12 \%$ de adesivo não diferiram significativamente dos painéis com de $8 \%$ e $10 \%$ de UF. Apenas os painéis com $8 \%$ de adesivo atenderam a norma ANSI/A 208.1 (American National Standard, 1993) que estabelece valores mínimos de 1,0 kgf/cm², para essa propriedade.

Os autores Maciel et al. (2004a), trabalhando com painéis produzidos a partir de $50 \%$ de PS e $50 \%$ de Pinnus elliotti, com proporções de $4 \%$ e $6 \%$ de adesivo (UF), encontraram valores superiores aos relatados nesse trabalho que foram, respectivamente de, 4,89 e 5,30 kgf/ $\mathrm{cm}^{2}$. 
Segundo Guimarães Júnior et al. (2016), a diferença observada entre os resultados do presente trabalho e os painéis de Pinnus elliotti se deve principalmente a baixa densidade dos resíduos de bagaço de cana, pois o aumento no número de partículas acarreta em menor disponibilidade de adesivo por partícula, o que prejudica a colagem.

Na Figura 10, observa-se a relação entre MOEd e MOE estático, para os painéis produzidos com as diferentes proporções de adesivo e PS. Entre as equações testadas para estimar o MOE estático a partir do MOEd, a equação a seguir foi escolhida por apresentar o maior $\mathrm{R}^{2}$, de 0,8051 , um dos menores erros, de $181,64 \mathrm{kgf} / \mathrm{cm}^{2}$ e o valor de $\mathrm{F}$ significativo, de 110,41 .

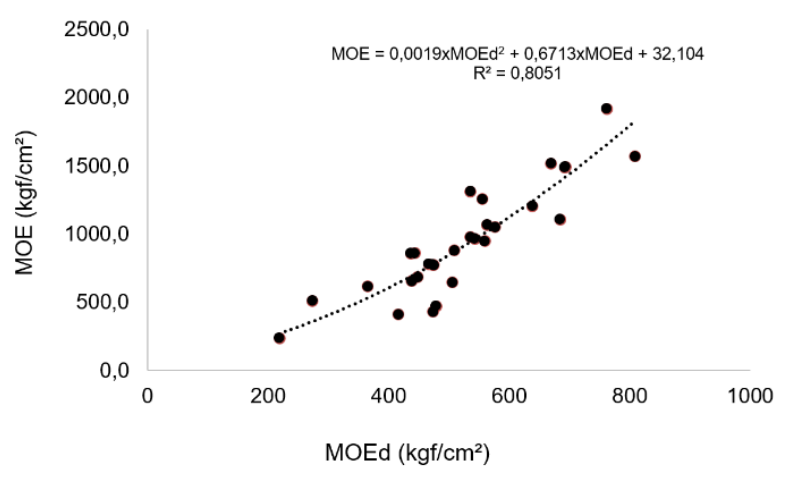

Figura 10. Estimativa do MOE em função do MOEd. *Médias seguidas pela mesma letra não se diferem estatisticamente pelo teste Tukey, a 5\% de significância.

Uma das principais vantagens em se utilizar o MOEd para estimar o MOE estático referese à possibilidade de realizar ensaios não destrutivos, ao invés de aplicar ensaios destrutivos, os quais são demorados e trabalhosos, e exigem um grande número de amostras (Mendes et al., 2012b).

Os autores Mendes et al. (2012b), avaliando o módulo de elasticidade de painéis aglomerados de bagaço de cana através do MOEd, verificaram elevada confiabilidade do método de estimativa do MOE estático através de um alto valor de $\mathrm{R}^{2}$ que foi de 0,976 , valor próximo ao relatado neste trabalho.

\section{CONCLUSÕES}

Os painéis foram classificados como sendo de baixa densidade, podendo ser indicados em aplicação de isolamento térmico acústico após a realização de testes, decoração entre outros que não exijam elevada solicitação de esforço mecânico.

A adição de PS proporcionou melhoria nas propriedades físicas de teor de umidade, absorção de água e inchamento em espessura dos painéis. Já para as propriedades mecânicas o PS não afetou significativamente tendo em vista que não houve diferença significativa entre os painéis com $0 \%, 20 \%$ e $30 \%$ de PS.

As diferentes porcentagens de adesivo tiveram baixa influência nas propriedades físicas dos painéis, entretanto, nas propriedades mecânicas quando se adicionou $10 \%$ de adesivo houve uma redução no MOR, MOE e tração perpendicular, quando comparado com 8 e $12 \%$.

A partir do MOEd foi possível ajustar uma equação com um alto valor de $R^{2}$ para a estimativa do MOE estático.

Com base nos dados obtidos, recomenda-se adição de 30\% de PS, 70\% de bagaço de cana e $8 \%$ de adesivo UF para a confecção dos painéis de bagaço de cana.

Recomenda-se a realização de pesquisas que envolvam tratamento químico das partículas de bagaço de cana antes da confecção dos painéis e fazer o uso de emulsão de parafina para melhorar as propriedades físicas como teor de umidade, absorção de água e inchamento em espessura dos painéis. 


\section{REFERÊNCIAS BIBLIOGRÁFICAS}

Alonge, F. A., Chamma, P. V. C., \& Rocha, R. R. (2014). Produtos ecoeficientes na arquitetura: a produção de painéis feitos a partir do compósito de plástico e casca de arroz. Periódico Eletrônico Fórum Ambiental da Alta Paulista, 10(1), 13-27.

American National Standard - ANS. (1993). ANSI/A 208.1.1993: matformed wood particleboard: specification (9 p.). Gaithersburg: National Particle Boards Association.

American Society for Testing and Materials - ASTM. (1998). D 1084-97: standard test methods for viscosity of adhesives. West Conshohocken: ASTM Internacional.

American Society for Testing and Materials - ASTM. (1999). D 2471-99: standard test method for gel time and peak exothermic temperature of reacting thermosetting resins. West Conshohocken: ASTM Internacional.

American Society for Testing and Materials - ASTM. (2001). D3878-01: standard terminology for composite materials. West Conshohocken: ASTM International.

American Society for Testing and Materials - ASTM. (2013). D 1490-01: standard test method for nonvolatile content of urea-formaldehyde resin solutions (2 p.). West Conshohocken: ASTM Internacional.

American Society for Testing and Materials - ASTM. (2015). E70-07: standard test method for ph of aqueous solutions with the glass electrode. West Conshohocken: ASTM Internacional.

Associação Brasileira de Produtores de Florestas Plantadas - ABRAF. (2010). Retrieved in 2018, June 12, from http://www.ipef.br/estatisticas/relatorios/anuario-ABRAF11-BR.pdf

Associação Brasileira de Normas Técnicas - ABNT. (2002). NBR 14810-3: chapas de madeira aglomerada: métodos de ensaio (32 p.). Rio de Janeiro: ABNT.

Baldin, T., Silveira, A. G., Vidrano, B. R. A., Cancian, L. C., Spatt, L. L., \& Haselein, C. R. (2016). Qualidade de painéis aglomerados produzidos com diferentes proporções de madeira e capim-annoni. Agrária, 11(3), 230-237. http://dx.doi.org/10.5039/agraria.v11i3a5376.

Banco Nacional de Desenvolvimento Econômico e Social - BNDS. (2002). Aspectos gerais do poliestireno. BNDES Setorial, 16, 123-136.

Bedin, M. G. (2009). Compósito com polietileno de baixa densidade e fibra de coco in natura e modificada (Dissertação de mestrado). Universidade Estadual do Norte Fluminense Darcy Ribeiro, Campos dos Goytacazes.

British Standards Institution - BSI. (1993). EN 310-93: wood-based panels: determination of modulus of elasticity in bending and of bending strength. London: BSI.

Caraschi, J. C., Leão, A. L., \& Chamma, P. V. C. (2009). Avaliação de painéis produzidos a partir de resíduos sólidos para aplicação na arquitetura. Ciência e Tecnologia, 19(12), 47-53. http://dx.doi.org/10.1590/S0104-14282009000100012.

Carvalho Neto, A. G. V., Ganzerlli, T. A., Fávaro, S. L., Suguino, B. S., Carvalho, G. M., \& Radovanovic, E. (2009). Preparação e caracterização de compósitos de polietileno pós-consumo reforçados com fibras do bagaço de cana. In Anais do $10^{\circ}$ Congresso Brasileiro de Polímeros, Foz do Iguaçu, PR.

Carvalho, L. H., \& Cavalcanti, W. S. (2006). Propriedades mecânicas de tração de compósitos poliéster/tecidos híbridos sisal/vidro. Polímeros. Ciência e Tecnologia, 16(116), 33-37.

Cloutier, A. (1998). Oriented stranboard (OSB): raw material, manufacturing process, properties of wood-base fiber and particle materials. In International Seminar on Solid Wood Products of High Technology (pp. 173-185). Belo Horizonte: SIF.

Commercial Standard. (1968). CS 236-66: mat formed wood particleboard. Geneva.

Companhia Nacional de Abastecimento - CONAB. (2018). Retrieved in 2018, May 8, from https://www.conab.gov.br

Geyer, R., Jambeck, J. R., \& Law, K. L. (2017). Production, use, and fate of all plastics ever made. Science Advances, 3(7), e1700782. PMid:28776036. http://dx.doi.org/10.1126/sciadv.1700782.

Gouveia, E. R., Nascimento, R. T., Souto-Maior, A. M., \& Rocha, G. J. M. (2009). Validação de metodologia para a caracterização química de bagaço de cana-de-açúcar. Quimica Nova, 3(6), 1500-1503. http://dx.doi.org/10.1590/S0100-40422009000600026.

Guimarães Júnior, J. B., Xavier, M. M., Santos, T. S., Protásio, T. D. P., Mendes, R. F., \& Mendes, L. M. (2016). Inclusão de resíduo da cultura de sorgo em painéis aglomerados de eucalipto. Pesquisa Florestal Brasileira, 36(88), 435-442. http://dx.doi.org/10.4336/2016.pfb.36.88.1036. 
Indústria Brasileira de Árvores - IBÁ. (2017). Relatório anual 2017. Retrieved in 2018, May 8, from http://iba.org/images/shared/Biblioteca/IBA_RelatorioAnual2017.pdf

Iwakiri, S. (2005). Painéis de madeira reconstituída. Curitiba: FUPEF.

Iwakiri, S., Shimizu, J., Silva, J. D. C., \& Menezzi, C. S. (2004). Produção de painéis de madeira aglomerada de Grevillea robusta A. Cunn. ex R. Br. Revista Árvore, 28(6), 883-887.

Kelly, M. W. (1977). A critical literature review of relationship between processing parameters and physical properties of particleboard (Forest Products Laboratory General Technical Report, No. 10, pp. 1-66). Madison: USDA, Forest Service.

Luz, S. M., Gonçalves, A. R., \& Del'Arco Junior, A. P. (2006). Microestrutura e propriedades mecânicas de compósitos de polipropileno reforçado com celulose de bagaço e palha de cana. Matéria, 11(2), 101-110. http://dx.doi.org/10.1590/S1517-70762006000200004.

Maciel, A. S., Vital, B. R., Della Lucia, R. M., \& Pimenta, A. S. (2004a). Chapas de madeira aglomerada produzidas com partículas de Eucalyptus grandis w. Hill exmaiden, poliestireno (PS) e polietileno tereftalato (PET). Cerne, 10(1), 53-66.

Maciel, A. S., Vital, B. R., Della Lucia, R. M., \& Pimenta, A. S. (2004b). Painéis de partículas aglomeradas de madeira de Pinus elliottii Engelm., poliestireno (PS) e polietileno tereftalato (PET). Revista Árvore, 28(2), 257-266.

Mano, E. B., \& Mendes, L. C. (1999). Introdução a polímeros (2. ed.). São Paulo: Edgard Blücher.

Mendes, R. F., Mendes, L. M., Carvalho, A. G., Guimarães Júnior, J. B., \& Mesquita, R. G. A. (2012a). Determinação do módulo de elasticidade de painéis aglomerados por Stress Wave Timer. Floresta e Ambiente, 19(2), 117-122. http://dx.doi.org/10.4322/floram.2012.013.

Mendes, R. F., Mendes, L. M., Guimarães Júnior, J. B., Santos, R. C., \& César, A. A. S. (2012b). Efeito da associação de bagaço de cana, do tipo e do teor de adesivo na produção de painéis aglomerados. Ciência Florestal, 22(1), 161-170. http://dx.doi.org/10.5902/198050985088.

Minas Gerais. (2009). Plano de gerenciamento integrado de resíduos plásticos - PGIRP. Belo Horizonte: Fundação Estadual do Meio Ambiente, Fundação Israel Pinheiro.

Moslemi, A. A. (1974). Particleboard: materials (Vol. 1, 244 p.). London: Southern Illinois University.

Pedreschi, R. (2009). Aproveitamento do bagaço de cana da indústria sucroalcooleira na produção de painéis aglomerados (Tese de doutorado). Universidade Federal de Lavras, Lavras.

Sanchez, E. M. S., Cavani, C. S., Leal, C. V., \& Sanchez, C. G. (2010). Compósito de resina de poliéster insaturado com bagaço de cana-de-açúcar: influência do tratamento das fibras nas propriedades. Polímeros, 20(3), 194-200. http://dx.doi.org/10.1590/S0104-14282010005000034.

Santos, R. C., Mendes, L. M., Mori, F. A., \& Mendes, R. F. (2009). Chapas de partículas aglomeradas produzidas a partir de resíduos gerados após a extração do óleo da madeira de candeia (Eremanthus erythropappus). Scientia Forestalis, 37(84), 437-446.

Soares, S. S., Guimarães Júnior, J. B., Mendes, L. M., Mendes, R. F., Protásio, T. P., \& Lisboa, F. N. (2017). Valorização do bagaço de cana-de-açúcar na produção de painéis aglomerados de baixa densidade. Ciência da Madeira, 8(2), 64-73. http://dx.doi.org/10.12953/2177-6830/rcm.v8n2p64-73.

Spinacé, M. A. S., \& De Paoli, M. A. (2005). A tecnologia da reciclagem de polímeros. Química Nova, 28(1), 65-72. http://dx.doi.org/10.1590/S0100-40422005000100014.

Technical Association of the Pulp and Paper Industry - TAPPI. (1997). TAPPI T $264 \mathrm{~cm}$-97: preparation of wood for chemical analysis. Atlanta: Tappi Press.

União da Indústria de Cana-de-Açúcar - UNICA. (1996). Retrieved in 2018, May 8, from http://www.unica.com.br/faq/

$\mathrm{Wu}, \mathrm{Q}$. (1999). In-plane dimensional stability of oriented strand panel: effect of processing variables. Wood and Fiber Science, 31(1), 28-40.

Contribuição dos autores: KBF: conceituação, curadoria de dados, análise formal, investigação, escrita - primeira redação; JCF: conceituação, análise formal, metodologia, administração do projeto, recursos, supervisão, escrita revisão e edição; CRA: conceituação, análise formal, recursos, supervisão, escrita - revisão e edição; DET: metodologia, recursos, supervisão; LFAG: metodologia, recursos, supervisão; ÍLG: visualização, escrita - revisão e edição; TES: Investigação; DKF: investigação. 